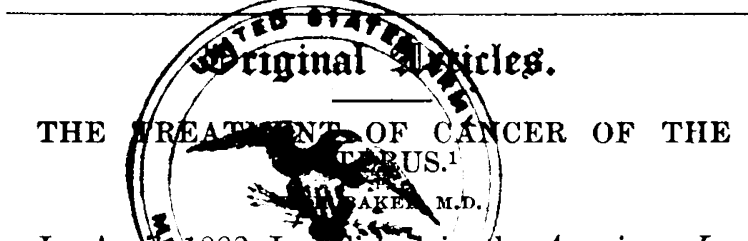

IN Aprtit 882, I published in the American Journal of Obstercics, the description of an operation for cancer of the whisenters proved to give better results than any oteperem of high amputation, and which has been adopted by other surgeons with a like satisfactory result. As the steps of this operation are not well understood by the general surgeon, I have thought best to call the attention of the profession again to its description, which is as follows:

'The patient being etherized and placed in the Sims' position, the cervix is seized with the volsellum forceps and dragged down as nearly to the outlet as possible. This not only facilitates manipulation, but diminishes, in fact almost entirely checks, the hæmorrhage which otherwise may be alarming. The portio vaginalis is ther cut into anteriorly with the scissors, and the supravaginal cervix anteriorly is separated from the bladder with the scissors, aided by the forefinger in tearing the tissues. This part of the operation is similar to Schroeder's for the removal of the uterus by the vagina.

The same incision is then made into the vagina posteriorly, and the supravaginal cervix separated from the peritoneum up to the level of the internal os uteri. Thus it will be seen that the peritoneum is not purposely opened in an uncomplicated case, as is done by Schroeder's method. But the peritoneum is closely attached to the uterus at the level of the internal os, and it may accidentally be cut into, which has been my experience in two instances. The anterior and posterior incisions being now connected by lateral ones, and the supravaginal cervix separated on the sides in the same manner as was done in front and behind, the aterotome is to be substituted for the scissors, and a funnel-shaped portion of the body of the uterus cut out. This step of the operation is like Sims', with the exception that here it is possible to remove more of the body of the uterus, because here the base of the cone removed starts from the level of the internal os uteri at the junction of the peritoneum to the body of the uterus, both before and behind, and the apex of the cone removed extends nearly or quite to the fundus of the uterus; whereas, following Sims' method, the base of the cone starts from the level of attachment of the vagina to the cervix, and a portion of the supravaginal cervix and more of the body of the uterus must be left.

It is thus possible to remove the entire cervix, both infra- and supra-vaginal, and at the same time nearly or quite one-half of the body of the uterus, and that the most important half ; for cancer of the body of the uterus, whether of primary origin or of extension from the cervix affect the glandular structure first, and by the method I have described, this, its natural habitat, is removed or destroyed. It is in the connective tissues which so predominate in the cervix that the disease there develops so readily, and this, it has been seen, is entirely removed. The actual cautery, at a red heat, is then applied to the whole denuded surface. This part of the operation takes considerable time ; for, as the traction is relaxed, there is likely to be more or less hæmor-

1 Read before the Obstetrical Section of the Suffolk District Medi ael Society, February 17, 1886.

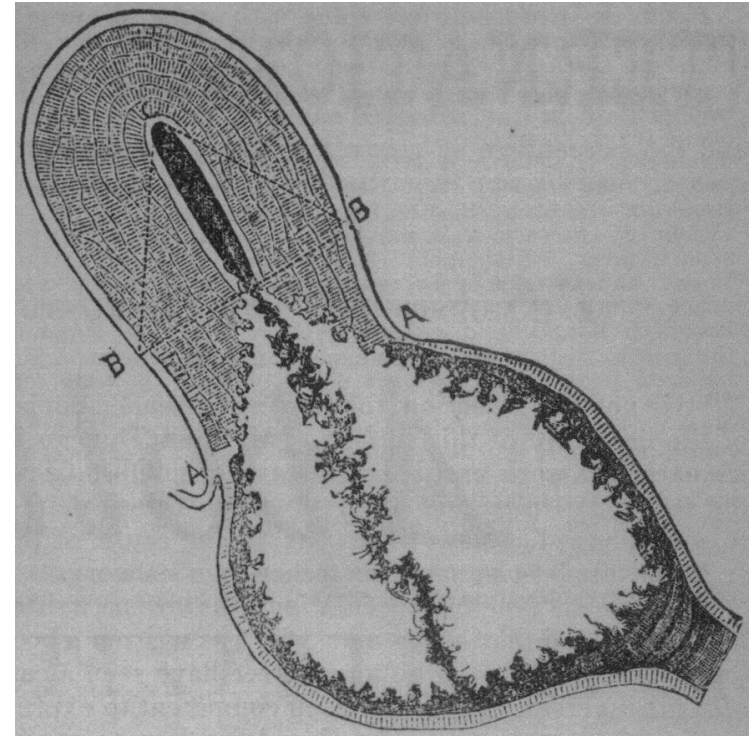

rhage, and it often requires much patience to apply the cautery thoroughly enough to each and every bleeding point to feel secure in putting the patient to bed without tampon or other dressing to control hæmorrhage. It is possible to accomplish this, however, and it is a very great gain in the subsequent treatment of the patient. The operation can seldom be done in less than half an hour, and will frequently require from three-quarters of an hour to an hour.

So long as the disease has not touched the rectum, we may still hope to remove it entirely, as I have done in several instances, even though it has implicated the whole thickness of the vaginal membrane and submucous tissue quite to the peritoneum posteriorly over a large surface. The operation must differ from that just described, in that the incision into the vagina posteriorly must be made a full quarter of an inch beyond the line of any infiltration, and extend quite through into the peritoneal cavity, removing the whole of the upper vagina with the supravaginal cervix. Before applying the cautery, the edge of the healthy vagina should be stitched with silver sutures to the lower part of the posterior wall of the uterus. So too, if the anterior vaginal membrane be implicated in the disease, it may be dissected off the bladder. If the base of the bladder itself even be affected, so long as the ureters are not involved, we may cut away a large part of it, if we can thereby remove the entire disease, closing the fistula thus formed with silver wire before using the cautery.

The advantages claimed for this method of operating are :

First, That by it we are able to remove more of the uterus than by any other form of high amputation.

Second, The opening of the peritoneal cavity is not necessarily involved in its performance.

Third, The practicability of using the touch in determining the extent of the disease as the operation proceeds, is retained, which cannot be practiced when the galvano-caustic wire is used.

Fourth, All the advantages of the galvano cautery are retained by the application of the Thermo cautery at a red heat, to all the denuded surface, and made more effectual, even, by previously being sure that the disease as evidenced by the touch had been removed. 
Fifth, It is more practicable for the general surgeon than total extirpation.

Sixth, The length of respite from the disease is greater than in any reported cases of total extirpation and the percentage of recoveries from the operation greater than by any other method of high amputation.

\section{ACUTE INVERSION OF THE UTERUS. ${ }^{1}$}

BY A. P. WEEKS, M.D.

IT is not my intention to give an extended review of the literature of this subject. Matthew Duncan, in his excellent work entitled " Researches in Obstetrics," did that very fully and ably. Nor do I desire either to put forward a new theory as to the cause of this accident, or to bolster up any other man's theory.

Too much fanciful speculation has been written already. I find that every man who has written a book on obstetrics, although he may never have seen a case of acute inversion, has felt himself competent to express opinions concerning it, and to lay down laws to govern other men's practice in the management of it.

As the observations noted in my case do not harmonize with the teachings of many of our systematic writers on obstetrics, I have collected as many histories of cases as my time would admit, which, together with my own case, I wish carefully to review, in order that we may see what experience teaches of this dangerous accident.

REPORT OF MY CASE.

Ḿrs. M- aged twenty-two, primipara; rather anæmic; never strong; of slight build, but wellformed; at full term; without previous pain, had the membranes rupture about seven o'clock on the morning of the 23d of June, 1883. She had no pains worth noticing until late in the evening following. About three o'clock in the morning of the $23 \mathrm{~d}$, that is, twentyone hours after the escape of the waters, I was called. I found the os just commencing to dilate. The head was presenting, and I could distinguish the position, O. L. A. The pains were sufficiently frequent, but short. I did not remain with the patient, but called in frequently during the forenoon to watch the progress of the case.

About noon, I went to the house to remain till the completion of the labor. At, or near one o'clock, the head had descended so as to rest on the perineum, but had not escaped from the os. The pains now, instead of growing stronger and more effectual, became weak and almost ineffectual. At half-past two, there had been no advancement for an hour. I then applied the forceps without ether, and easily delivered an eight and three quarters-pound child without injury to the mother. The nurse compressed the abdomen after the delivery of the head until the child was fully expelled. When I had cared for the child and cleansed my hands, I took the nurse's position and held the uterus in hand several minutes, until I felt contraction; then I steadied the cord, but did not draw upon it. I continued the compression with the left hand, and told the woman to bear down. She did so once, and only once. I noticed that the globular form of the uterus became less distinct. I could still feel it, but I did not think to trace its outline carefully. Still holding $\mathrm{my}$ hand on the abdomen,

1 Read before the Obstetrical Section of the Suffolk District Medical Society, February $17,1886$.
I passed the right index finger into the vagina, and found the placenta hąd descended.

As I could not reach the border of it, I pierced it and rotated it, when it was quickly expelled. Quite a brisk hæmorrhage followed. I passed two fingers to explore the vagina, seeking the cause of bleeding. I found a large tumor, filling nearly down to the perineum. The uterus had inverted. I at once passed the whole hand into the vagina, grasping the fundus, and compressing it evenly over all sides, and still supporting the abdomen with my left hand, I gently carried the organ to its place. It took some two or three minutes to accomplish it. Ergot was administered, and the hand retained in the cavity, and ice applied externally until a firm contraction was secured.

Now, as I introduced my hand, this was what I observed. A pear-shaped body, that filled my whole hand, was projecting into the vagina. I carried my fingers up, to learn that the inversion was not complete. I could detect on either side, the thick, rough walls of the uterus as compared with the thin, smooth, yielding walls of the vagina. There appeared to be about one and $a$ half to two inches of the uterus, about the length of the natural neck, not yet inverted. Holding my fingers well pressed up, to prevent further descent, with my thumb and little finger, which I was obliged to quite fully extend, I searched for the os. I could just distinguish it. It seemed like a mere thickened band at the vault of the vagina, perfectly flabby and inert. I fretted it, but it would not contract.

I then proceeded to carry the fundus to place by compressing, and pushing upward at the same time, replacing in an inverse order from the descent. The last to go up was the fundus; and this, be it remembered, was not flaccid, paralyzed, as some assert, but went up with a snap, and immediately grasped the fingers. The firmest contraction was at the fingers' tips. I could distinguish the compression as far down as the knuckles. I now felt for the os again. I felt it more plainly. It was distended, and evinced no disposition to contract. The uterus, for several minutes after the re-position, was of the shape of an inverted tunnel. The fundus was firmly contracted, the upper segment partially so, while the lower segment, cervix, and os, were inert.

I cannot tell the exact time required to arouse contractions of the os; I think more than fifteen minutes. At the expiration of a half-hour, the cervix had not assumed the elongated form that it afterwards showed. The lady made a very rapid and gratifying recovery. She did not have one unpleasant symptom, not so much as gaseous distention, so common after normal labors, and to-day she experiences no uterine weakness or illfeeling as a consequence of the accouchement.

I have described thus minutely, every condition and procedure attending this labor, to show that inversion of the uterus may occur without there being present, a rapid delivery, short funis, traction of the funis, irregular compression of the fundus, or undue bearingdown efforts of the patient - factors which most obstetric authors enumerate and emphasize as causes of the accident. So much of experience - now what does it teach us?

We have no statistics from which to make comparisons to determine the relative frequency of this accident. I think, however, that it is not so rare as it has heretofore been supposed. The ratio, 1 to 190,800 , as observed in the Rotunda Hospital, which we find quoted in nearly every work on obstetrics, does not, in 